\title{
A flexible and low power telemetric sensing and monitoring system for chronic wound diagnostics
}

\author{
Nasir Mehmood $^{1 *}$, Alex Hariz ${ }^{1}$, Sue Templeton ${ }^{2}$ and Nicolas H Voelcker ${ }^{3}$
}

\author{
* Correspondence: nasir. \\ mehmood@unisa.edu.au \\ ${ }^{1}$ School of Engineering, University \\ of South Australia, Adelaide, SA, \\ 5001, Australia \\ Full list of author information is \\ available at the end of the article
}

\begin{abstract}
Background: Non-healing chronic wounds, such as venous leg ulcers, can be monitored non-invasively by using modern sensing devices and wireless technologies. The development of such a wireless diagnostic tool may improve chronic wound management by providing evidence on efficacy of treatments being provided. This paper presents a low-power portable telemetric system for wound condition sensing and monitoring. The system aims at measuring and transmitting real-time information of wound-site temperature, sub-bandage pressure and moisture level from within the wound dressing.

Methods: Commercially available non-invasive temperature, moisture, and pressure sensors are interfaced with a telemetry device on a flexible $0.15 \mathrm{~mm}$ thick printed circuit material to construct a light-weight, non-invasive, biocompatible, and low-power sensing device. The real-time data obtained is transmitted wirelessly to a portable receiver which displays the measured values. The performance of the whole telemetric sensing system is validated on a mannequin leg using commercial compression bandages and dressings. A number of trials on a healthy human volunteer are performed where treatment conditions were emulated using various compression bandage configurations.

Results: A reliable and repeatable performance of the system is achieved under compression bandage and with minimal discomfort to the volunteer. The system is capable of reporting instantaneous changes in bandage pressure, moisture level and local temperature at wound site with average measurement resolutions of $0.5 \mathrm{mmHg}$, $3.0 \% \mathrm{RH}$, and $0.2^{\circ} \mathrm{C}$ respectively. Effective range of data transmission is $4-5 \mathrm{~m}$ in an open environment.
\end{abstract}

Conclusions: A flexible and non-invasive sensing system is developed to acquire and wirelessly transmit wound parameters from within a compression bandage and wound dressing worn on a human limb. Pre-clinical results on a healthy human subject suggest its clinical usability and value to health practitioners. However, further performance evaluations of the device on a wider population of healthy human subjects and on patients with chronic wounds are required to confirm its medial usefulness and to quantify its real impact on chronic wound management.

Keywords: Chronic wound monitoring, Telemetric sensing, Chronic wound management, Wound diagnostic system

\section{Introduction}

Chronic wounds, such as venous leg ulcers and diabetic foot ulcers, are posing a financial threat to the healthcare systems in the world $[1,2]$. A current estimate shows the economic cost of woundcare activities in the world is distributed as $15-20 \%$ materials,

(C) 2015 Mehmood et al.; licensee BioMed Central. This is an Open Access article distributed under the terms of the Creative Commons Attribution License (http://creativecommons.org/licenses/by/4.0), which permits unrestricted use, distribution, and reproduction in any medium, provided the original work is properly credited. The Creative Commons Public Domain Dedication waiver (http://creativecommons.org/publicdomain/zero/1.0/) applies to the data made available in this article, unless otherwise stated. 
$30-35 \%$ nursing time and more than $50 \%$ as hospitalization time [3]. The woundcare cost in Australia only is estimated at \$ 2.5 billion per year with more than 4000 limb amputations [4]. A 2001 study indicated that chronic wounds were a major cause of morbidity, affecting more than $1 \%$ of UK population and with treatment cost of at least $£ 1$ billion [5]. During 2006-07, chronic wounds were affecting 3-6 million people in the USA with a total cost of treatment estimated at more than $\$ 3$ billion annually $[6,7]$. In 2012, approximately 7 million people suffered from chronic wounds in the USA, and the cost for their treatment was estimated at almost \$25 billion annually [8].

The most effective and economical treatment of wounds is covering them with a suitable dressing or bandage in order to protect damaged skin from external effects such as microorganism attacks [9]. For certain chronic wounds such as venous leg ulcers, appropriate compression bandages are applied to increase the healing rate [10,11]. These bandages may be retention (low pressure), light support (medium pressure) or compression (high pressure) bandages. The method of applying compression bandages on the affected limb is very important as the efficacy and maintenance of sub-bandage pressure depends on it [12]. Compression bandages can produce a pressure up to $60 \mathrm{mmHg}$ at the ankle (extra high pressure), while the recommended high pressure value is $40 \mathrm{mmHg}$ at the ankle [10,12]. Depending on the applied pressure range and the type of bandage used, the sub-bandage pressure may vary significantly during the physical movement of the patient, thus affecting the healing rate [13]. In addition to compression bandages, healing rates may also be increased by managing moisture produced by the wound (exudate) through moisture-retentive dressings such as Anasept (hydrogel) and Hydrocolloids [14,15] for wounds with low exudate and dressings such as Allevyn (foam) or Melgisorb ${ }^{\circ}$ (calcium alginate) for wounds with moderate to high exudate. In addition to moisture levels, the temperature and $\mathrm{pH}$ under the dressing may change as a result of an infection [16,17]. Unfortunately, these parameters associated with the dressings are not currently monitored in clinical practice. There is an opportunity for advanced sensor technologies to contribute to improved wound monitoring and diagnostics.

A highly accurate $\left(0.2^{\circ} \mathrm{C}\right.$ accuracy) RFID-based skin temperature monitoring system was devised by Matzeu et al. [18]. However, the system lacked proper clinical trials using wound dressings. Moser and Martin [19] presented a flexible platinum-based miniaturized temperature sensor to operate within $0-400^{\circ} \mathrm{C}$ range. For this device too, no testing on humans was performed. McColl et al. [20] developed an impedance sensor moisture monitoring system to be used with wound dressings. Following this, Ohmedics $^{\odot}$ developed a clinically-proven moisture monitoring device called WoundSense $^{\circ}$ [21]. However, this device is not designed to stay within the dressing for continuous moisture sensing. Khaburi et al. [22] reported a force sensors-based pressuremapping bandage prototype to measure pressure at various points on a leg mannequin. A wearable sub-bandage pressure measurement system is reported by V. Casey et al. [23] for real-time pressure measurements with a wired connection between the flexible sensor and the display module. To the best of our knowledge, no device exists for continuous and non-invasive monitoring of sub-bandage pressure for wounds. However, miniaturised pressure sensors have been fabricated and used in other applications including intracranial pressure [24,25], intraocular pressure [26], spinal plates pressure [27] and for general in vivo applications [28-30]. In other reported literature, 
researchers have proposed integrated wound monitoring systems using wireless data transmission [31,32]. However, those devices have not been tested or utilised in a wound environment and their wireless transmission range was confined to a few millimetres, thus restricting the practical use of the devices.

In this paper, we demonstrate a flexible wireless telemetric system for continuous sensing and monitoring of the wound environment, proposed in our earlier review article [33]. Preliminary results indicate that the system is capable of measuring and transmitting real-time information on temperature, moisture, and sub-bandage pressure from under the bandage or within the wound dressing at programmable transmission intervals [34]. The selection of sensors and their calibration processes have been discussed in our recent article [35]. The sensing system is fabricated on a flexible printed circuit material, while the sensors are micro-sized and flexible, thus making the system minimally invasive to wounds and the human body. The receiver is portable with the capability to receive data accurately within a distance of 4-5 meters. The system has been tested on a human volunteer using various compression bandages and moistureretentive dressings. The results from these trials confirm the clinical utility of this system in a wound environment.

\section{Methods and materials}

For chronic wound monitoring application, the diagnostic device is required to perform reliably in a delicate environment involving human skin and wound fluid. In addition to satisfy the essential criteria of flexibility, protection from wound chemicals, and biocompatibility, the device needs to fulfil certain performance requirements as well, which sets the foundation for minimum measurement resolutions. For meaningful temperature measurements, the device must be able to detect changes in temperature of less than $\pm 0.5^{\circ} \mathrm{C}$. The case with pressure and moisture is different. Although, the aim of compression bandages and stockings is to maintain a constant sub-bandage pressure at certain positions on limb, however, it may be anticipated that a $\pm 5 \mathrm{mmHg}$ variation in bandage pressure would not have a significant impact on wound healing. Similarly, a $\pm 5 \% \mathrm{RH}$ resolution could be expected for moisture measurements. The wireless device for this application does not need to transmit continuously as the wound conditions do not change abruptly. A complete packet of information transmitted twice an hour would be sufficient. All the user and medical requirements for the wound sensing system are listed in Table 1 . The proposed sensing system satisfies all of the above mentioned medical and performance requirements as explained in the following sections.

\section{Selection and calibration of sensors}

For wound monitoring application, the sensors and their assemblies need to be biocompatible and minimally invasive to the human body, as the sensors would be placed within a wound dressing or compression bandage over a human limb [36]. Having metallic inflexible structures of sensors and circuit components would create discomfort to the patients. It has been revealed through online surveys that the majority of available sensors do not qualify for this particular application because of their large size, invasive structure, complex principle of measurement, and the need for additional on- 
Table 1 Requirement specifications for the developed chronic wound monitoring system

\begin{tabular}{lll}
\hline Parameters & Type/category & Specifications \\
\hline Sensors & Temperature & At least 01 sensor, $0.5^{\circ} \mathrm{C}$ resolution, miniature and flexible \\
& Moisture & At least 01 sensor, $5 \% \mathrm{RH}$ resolution, miniature and flexible \\
& Pressure & At least 01 sensor, $5 \mathrm{mmHg}$ resolution, miniature and flexible \\
System size & NA & Small enough for placement within a compression bandage \\
Power consumption & NA & Must be kept as low as possible for long-term operation \\
Transmission & Protocol & Any suitable protocol e.g. Zigbee ${ }^{\circ}$, Bluetooth, WiFi \\
& Range & Suitable to receive data in a clinical setup \\
& Power & Within the safe exposure limits for human body \\
& Frequency & 2.4 GHz band to keep the components' size smaller \\
Ergonomics & Interval & Should be kept high to save battery power, e.g. 10 minutes \\
Response time & NA & Must be flexible, bio-compatible, and non-invasive \\
\hline
\end{tabular}

board circuit components for operation. Table 2 below lists a few such sensors along with the reasons of their unsuitability for wound diagnostics.

Sensors need to be carefully selected, calibrated and characterized for the intended environment in order to capture reliable information. Any error in the sensor's measurement would spread through the whole system and in some scenarios might get amplified. This would create ambiguities and false diagnosis by clinicians and health practitioners. For wound-site temperature monitoring, we chose LM94021B (Texas Instruments, USA) temperature sensor for its small size and reliable performance. This ultra-low power sensor typically consumes just $9 \mu \mathrm{A}$ current at a rated $5.0 \mathrm{~V}$ supply voltage. With a size of $2.15 \mathrm{~mm} \times 2.40 \mathrm{~mm} \times 1.1 \mathrm{~mm}(\mathrm{~L} \mathrm{x} \mathrm{W} \mathrm{x} \mathrm{H})$ and a nominal accuracy of $\pm 1.5^{\circ} \mathrm{C}$ in the temperature range $20-40^{\circ} \mathrm{C}$ [37], the sensor is quite suitable for our wound monitoring system.

For moisture sensing, Honeywell HIH4030 piezo-electric and Multicomp's HCZ-D5 piezo-resistive moisture sensors were calibrated, characterized, and used with a prototype wireless sensing system. The piezo-resistive sensor (HCZ-D5) was found the most suitable sensor for this application because of its small size $(10 \mathrm{~mm} \times 5 \mathrm{~mm} \times$ $0.5 \mathrm{~mm}$ ). The sensor was calibrated and characterized using a dedicated experimental setup. An interface circuit was also designed to properly operate the moisture sensor.

For sub-bandage pressure measurement, we used the Interlink Electronics' FSR406 piezo-resistive pressure sensor with a square sensing area of $38 \mathrm{~mm} \times 38 \mathrm{~mm}$. This sensor is non-invasive, flexible and is only $0.5 \mathrm{~mm}$ in thickness. The pressure sensor was calibrated using a clinical-grade pressure meter HPM-KH-01 for validation of pressure measurements up to $40 \mathrm{mmHg}$ which is regarded as the desired value for high sub-bandage pressure [12]. An interface circuit was also designed to properly operate the pressure sensor. A commercial compression bandage system $\left(\right.$ Coban $\left.^{\text {Tw }} 2\right)$ was used to create pressure over the sensor placed on a mannequin leg.

\section{Flexible wireless sensing system}

A number of System-on-Chip (SoC) devices are commercially available with telemetry functions. Most of these devices use standard wireless transmission protocols, such as 
Table 2 A partial list of commercially available temperature, pressure and moisture sensors

\begin{tabular}{|c|c|c|c|}
\hline $\begin{array}{l}\text { Sensor } \\
\text { type }\end{array}$ & Model no. & Dimensions (mm) & Reasons of unsuitability \\
\hline Pressure & Honeywell 170PC & $21.5 \times 21.5 \times 34.3^{*}$ & $\begin{array}{l}\text { Invasive to human body due } \\
\text { to large size. Fluid flow is } \\
\text { required to create pressure. }\end{array}$ \\
\hline Pressure & $\begin{array}{l}\text { Honeywell } \\
\text { 19C015A7 }\end{array}$ & $19.0 \times 35.6^{\#}$ & $\begin{array}{l}\text { Invasive to human body due to } \\
\text { metallic port and large size. }\end{array}$ \\
\hline Pressure & $\begin{array}{l}\text { Measurement } \\
\text { Specialities }^{\text {TM }} \text { FC22 }\end{array}$ & $34.0 \times 16.8^{\#}$ & $\begin{array}{l}\text { Invasive to human body due to } \\
\text { large metallic structure. }\end{array}$ \\
\hline Pressure & $\begin{array}{l}\text { Measurement } \\
\text { Specialities }^{\text {TM MS5540C }}\end{array}$ & $6.4 \times 6.4 \times 3.0^{*}$ & $\begin{array}{l}\text { Invasive to human body due to } \\
\text { hard metallic structure. }\end{array}$ \\
\hline Pressure & Interlink Electronics FSR406 & $38 \times 38 \times 0.5$ & $\begin{array}{l}\text { Flexible, non-invasive, and } \\
\text { bendable structure. Works on } \\
\text { the principle of impedance } \\
\text { change with applied pressure. }\end{array}$ \\
\hline Moisture & Honeywell HIH403X/503X Series & $8.65 \times 4.20 \times 3.0^{*}$ & $\begin{array}{l}\text { Less invasive to human body } \\
\text { due to small size. Air samples } \\
\text { are required for moisture } \\
\text { measurement. }\end{array}$ \\
\hline Moisture & Silicon Laboratories Inc. Si7005 & $4.0 \times 4.0 \times 1.5^{*}$ & $\begin{array}{l}\text { Less invasive to human body } \\
\text { due to small size. Air samples } \\
\text { are required for moisture } \\
\text { measurement. Needs additional } \\
\text { on-board circuits to operate. }\end{array}$ \\
\hline Moisture & TDK Corporation CHS-SS Series & $25 \times 10 \times 5.0^{*}$ & $\begin{array}{l}\text { Invasive to human body due to } \\
\text { relatively large size. Air samples } \\
\text { are required for moisture } \\
\text { measurement. }\end{array}$ \\
\hline Moisture & Multicomp HCZ-D5 & $10 \times 5.0 \times 0.5^{*}$ & $\begin{array}{l}\text { Miniature size, comparatively } \\
\text { less invasive. } \\
\text { Works on the principle of } \\
\text { impedance change with } \\
\text { the moisture level. }\end{array}$ \\
\hline Temperature & Texas Instruments LM62 & $3.0 \times 1.4 \times 1.1^{*}$ & $\begin{array}{l}\text { Suitable for placement within } \\
\text { the dressing. Accuracy } \pm 3^{\circ} \mathrm{C} \text {. }\end{array}$ \\
\hline Temperature & Texas Instruments LM35DM & $6.5 \times 5.4 \times 2.0^{*}$ & $\begin{array}{l}\text { Dimensions are not suitable for } \\
\text { placement within the dressing. } \\
\text { Accuracy } \pm 0.5^{\circ} \mathrm{C} \text {. }\end{array}$ \\
\hline Temperature & Texas Instruments LM94021 & $2.15 \times 2.4 \times 1.1^{*}$ & $\begin{array}{l}\text { Dimensions suitable for placement } \\
\text { within the wound dressing. } \\
\text { Accuracy } \pm 1.5^{\circ} \mathrm{C} \text {. Gain can be } \\
\text { controlled digitally. }\end{array}$ \\
\hline
\end{tabular}

*Length $\mathrm{x}$ width $\mathrm{x}$ height in $\mathrm{mm}$, \# diameter $\mathrm{x}$ length in $\mathrm{mm}$.

These sensors are listed based on their available minimum size.

WiFi, Bluetooth ${ }^{\circ}$, Bluetooth Low Energy ${ }^{\circ}$, and ZigBee. Some examples of such SoCs are CC2530/31 (ZigBee), CC2540/41 (Bluetooth Low Energy), CC2560/64 (Bluetooth), CC3000/3100/3200 (WiFi), EM358x (ZigBee), 88MZ100 (ZigBee), ATMega128RFA1 (ZigBee) etc. In our proposed system, we have chosen ZigBee for its simplicity, reasonable range, and low power operations. For this purpose, we have used Atmel's ATMega128RFA1 RF transceiver in our sensing system [38]. The transceiver is of small size $(9 \mathrm{~mm} \times 9 \mathrm{~mm} \times 1 \mathrm{~mm}$ ) and operates at $2.45 \mathrm{GHz}$ ISM (industrial, scientific, medical) frequency band using IEEE 802.15.4 ZigBee protocol. It has a-100 dBm sensitivity and a $3.5 \mathrm{dBm}$ programmable output power. It also contains a programmable serial interface and a 10-bit analog-to-digital (ADC) converter, thus the hardware overhead would be minimized. 
For placement within the dressing, the wireless sensing system was designed and fabricated on a $0.15 \mathrm{~mm}$ 2-layer flexible printed circuit board (Figure 1(a)). In order to protect the delicate components within the bandage measurement environment, and to avoid any contact of circuit materials with the skin, a transparent biocompatible polydimethylsiloxane (PDMS) was coated over the exposed circuit components. The sensors were connected to port $\mathrm{F}$ (ADC input port) of the transceiver through the customised interface circuit. A balance-unbalance (Balun) device [39] (Johanson Technology ${ }^{\odot} \mathrm{P} / \mathrm{N}$. 2450FB15L0001 recommended for ATMega128RFA1) and a 2.4GHz chip antenna [40] (Johanson Technology ${ }^{\odot}$ P/N. 2450AT43B100) were used for accurate radio operations. Special care was taken while routing the RF circuit tracks and the RF components on the circuit board following the manufacturers' (Atmel ${ }^{\odot}$ and Johanson Technology ${ }^{\odot}$ ) guidelines. Exactly the same components and routing strategy were adopted on the receiver side. A firmware program was designed in $\mathrm{C}++$ and is implemented in the transceiver devices on both circuits i.e. transmitter (TX) and receiver (RX), using the Atmel's AVR Studio 6.0 software tool.

The information captured by the sensors was first converted into digital format and then stored into the TX frame buffer register. The first byte of TX frame buffer register was written with frame length information followed by the sensors' data [38]. Before transmitting, the transmitter was passed through a set of pre-defined states i.e. $\mathrm{RESET} \rightarrow \mathrm{TRX}$ _OFF $\rightarrow$ PLL_ON $\rightarrow$ TX_ON. After transmitting one packet of information, the transmitter was turned off (TRX_OFF) to save battery energy.

The net weight of the sensing system is $1.938 \mathrm{~g}$ without sensors, $6.709 \mathrm{~g}$ with all sensors attached and, $9.740 \mathrm{~g}$ with sensors and an alkaline battery having dimensions $14 \mathrm{~mm} \times 10 \mathrm{~mm}$ (length $\times$ diameter). The nominal and maximum current consumptions of the sensing system were measured as $13.58 \mathrm{~mA}$ and $17.4 \mathrm{~mA}$, respectively. Hence, the peak power consumption of the sensing system is $57.4 \mathrm{~mW}$ at $3.3 \mathrm{~V}$ supply

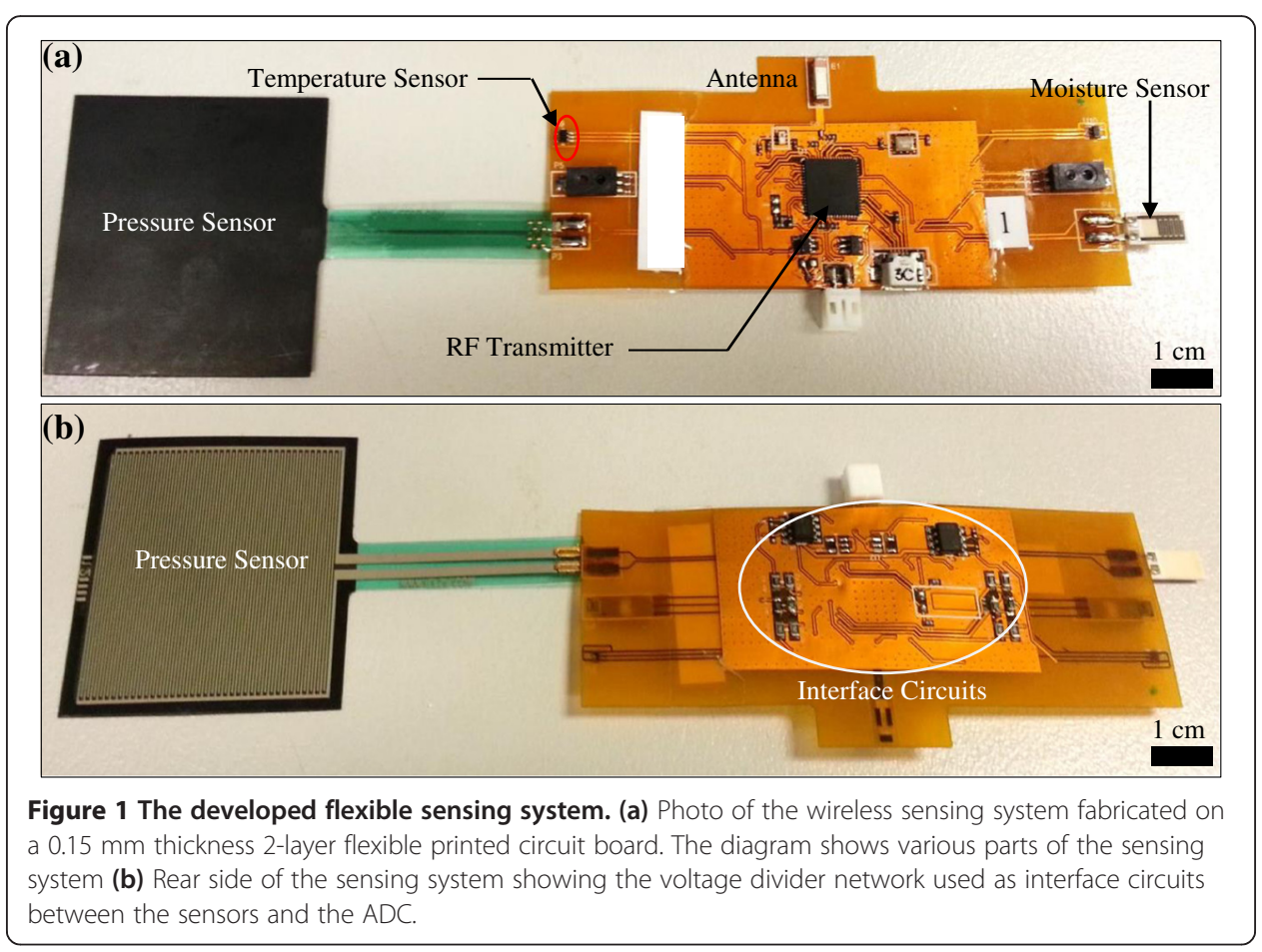


voltage. As most of the power is consumed in the RF circuits, reducing the RF switching frequency could save a considerable battery power. In a chronic wound monitoring application, even a very low data transmission rate (e.g. one packet of information per quarter an hour) would be sufficient because changes in wound parameters are very slow. A high data transmission rate would only mean a high switching rate of RF components resulting in unnecessary loss of battery power.

\section{Interfacing the sensors with the radio transmitter}

The result of ADC conversion was stored in two 8-bit registers; $\mathrm{ADCH}$ for high byte and ADCL for low byte. The maximum reference voltage for the ADC was $1.8 \mathrm{~V}$, which was internally generated and stabilized. All the sensors in our system work at a $5.0 \mathrm{~V}$ supply for all input values exceeding its reference voltage and would not be able to detect any input signal above $1.8 \mathrm{~V}$. This problem was resolved by reducing the ADC input voltage range such that the maximum input voltage of any sensor was less than the ADC reference voltage. For this purpose, a simple voltage divider circuit was designed using two surface mount resistors $R_{1}$ and $R_{2}$ for each sensor (Figure $1(b)$ ).

The voltage divider circuit necessitates a proper impedance matching with the sensors. The temperature sensor LM94021 consumes almost $9 \mu \mathrm{A}$ current at $5.0 \mathrm{~V}$. So, its impedance was nearly $555 \mathrm{k} \Omega$. In order to safely reduce the output voltage, we have chosen $750 \mathrm{k} \Omega$ standard values for both resistances in Figure 1(b) providing a voltage division ratio of two. The active moisture sensor HIH4030 operates at $5.0 \mathrm{~V}$, consuming almost $200 \mu \mathrm{A}$ of current, thus producing an input impedance of $25 \mathrm{k} \Omega$. For this sensor, we have used $R_{1}=46 \mathrm{k} \Omega$ and $R_{2}=25 \mathrm{k} \Omega$, resulting in a voltage division ratio of 2.84. Using the same principle for the pressure sensor FSR406, the resistance values were chosen as $R_{1}=56.2 \mathrm{k} \Omega$ and $R_{2}=46.4 \mathrm{k} \Omega$, resulting in a voltage division ratio of 2.21. For the passive moisture sensor HCZ-D5, the values of R1 and R2 were $46 \mathrm{k} \Omega$ and $25 \mathrm{k} \Omega$, respectively. The values of $R_{1}$ and $R_{2}$ might be adjusted to any other values provided they generate the same division ratio and their combined effect does not overrule the impedance matching criteria. It may be noted that the moisture level was measured with the passive sensor only.

\section{Information display module}

The transmitted data in ZigBee 802.15.4 protocol was received by the handheld receiver, processed by the T6963C LCD controller, and displayed on a $10 \mathrm{~cm} \times 8 \mathrm{~cm} \mathrm{LCD}$ screen [41]. The LCD controller was programmed through the receiver module (ATMega128RFA1) by sending the appropriate commands with required timings as recommended in the datasheet of T6963C [42]. The first transmitted byte consists of frame length information followed by the temperature, moisture and sub-bandage pressure data, respectively. Upon reception, the sensor's data was stored in a 128-byte frame buffer register. This data was then sent to the LCD controller (T6963C) through a parallel interface for processing and display. The LCD controller was also programmed to display the device ID (TX device), frequency channel (2405-2480 MHz in $5 \mathrm{MHz}$ steps), and received signal strength (i.e. RSSI) in $\mathrm{dBm}$.

The TX device ID may be used as a unique identifier for the patient under observation. In a hospital environment where a number of patients would be using these 
devices, unique frequency channels may be allocated to all devices to avoid any interference between devices or corruption of data. The RSSI level could be indicative of how trustworthy the information of measured parameters is. Information associated with much lower RSSI levels (e.g. $-90 \mathrm{dBm}$ ) may be discarded on the grounds that the TX device might be too far away from the RX or there might be some obstacle between them, thus inhibiting a reliable communication link. The experiments that involve a population sample for the purpose of evaluating the device from a clinical perspective are underway, they have received ethical approval, and will be the subject of a forthcoming paper. The experiments included in the present paper are carried out by a volunteer for the purpose of scientific evaluation of the device performance. They are not intrusive, and do not interfere with the bodily functions of the subject. They are no different than wearing a heart monitor or a blood-pressure monitor and recording the response as a function of activity. To that effect there is no ethical approval needed, and none were sought.

\section{Experiments and results}

The developed wireless wound sensing system was first tested in a room environment using commercial compression bandages and a mannequin leg. The compression bandages used for the experiments consisted of AMS Bi-Flex elastic bandage, Hartmann Lastodur Light long-stretch bandage, $3 \mathrm{M}$ Coban $^{\mathrm{rw}} 2$ two-layer, and Hartmann Lastolan short-stretch bandages. Adjunct components used in the application of compression bandage systems such as Soffban undercast padding and Idealcrepe crepe retention bandage were also used. Using the experimental setup, a number of experiments were performed using the mannequin leg in a room environment. The sensing system was placed flat on the central portion of the mannequin to avoid any damage to the components from bending. After the application of dressing, distilled water was sprayed over the bandage in the proximity of the moisture sensor. The measurements were taken with an interval of

Table 3 Results of initial experiments with the wireless sensing system in an open environment

\begin{tabular}{lllll}
\hline Time (minutes) & Energy $(\mathbf{d B m})^{*}$ & Temperature $\left(^{\circ} \mathbf{C}\right)$ & Moisture $(\% R H)$ & Pressure $(\mathbf{m m H g})$ \\
\hline T0 & -81 & 25 & 5 & 19 \\
T0 +5 & -81 & 24 & 3 & 18 \\
T0 +10 & -79 & 23 & 7 & 17 \\
T0 +15 & -79 & 23 & 9 & 17 \\
T0 +20 & -81 & 23 & 11 & 17 \\
T0 +25 & -78 & 23 & 12 & 16 \\
T0 +30 & -81 & 23 & 13 & 16 \\
T0 +35 & -79 & 23 & 14 & 16 \\
T0 +40 & -78 & 23 & 14 & 16 \\
T0 +45 & -81 & 23 & 16 & 16 \\
T0 +50 & -82 & 23 & 17 & 15 \\
T0 +55 & -79 & 23 & 17 & 15 \\
T0 +60 & -80 & 23 & 16 & 15 \\
T0 +65 & -80 & 23 & 16 & 15 \\
\hline
\end{tabular}

*Receiver sensitivity is $-100 \mathrm{dBm}$. Energy represents the energy detected during packet reception. 
5 minutes by the handheld receiver placed $3 \mathrm{~m}$ apart in line of sight to the wireless transmitter. The experiment was performed at $25^{\circ} \mathrm{C}$. The observations are reported in Table 3 using that the average values of received energy level, temperature, and sub-bandage pressure were calculated as $-79.92 \mathrm{dBm}, 23.21^{\circ} \mathrm{C}$ and $15.14 \mathrm{mmHg}$, respectively. The average errors in temperature, moisture, and sub-bandage pressure measurements were calculated as $0.93^{\circ} \mathrm{C}, 3.0 \% \mathrm{RH}$, and $1.50 \mathrm{mmHg}$, respectively. However, the error in sub-bandage pressure measurements is expected to increase on human leg because of flexible morphology and muscle movements. The errors in moisture and temperature measurements are immune to these factors.

The manufactured wound sensing and monitoring system (Figure 1) was extensively tested for its performance on a healthy volunteer. The objectives of these trials were to validate the performance of the sensing system under various clinical scenarios and to determine the optimum placement of the system and sensors on the human body. These scenarios were realized using different compression-bandage systems and various possible postures, both believed to influence the real-time measurements. These trials and their results are discussed in detail in the following sections.

\section{Verifying reliability of measurements}

In the first experiment the reliability of measurement results was tested, particularly the sub-bandage pressure measurements as they are prone to variations due to body movements. The sensing system was covered with a transparent adhesive silicone gel on both sides (Figure 2(a)). The trial was performed using a 4-layer compression system

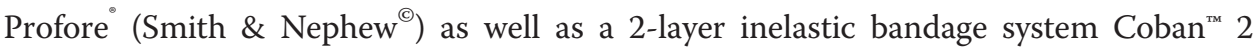
$\left(3 \mathrm{M}^{\odot}\right)$ for sub-bandage pressure measurements (Figure 2(b-c)). The bandages in all experiments were applied by a Wound Management Nurse Practitioner adept in managing chronic wounds and bandaging techniques. The sub-bandage pressure and moisture measurements were performed in two separate experiments. During the subbandage pressure measurements, the moisture sensor was placed directly on exposed
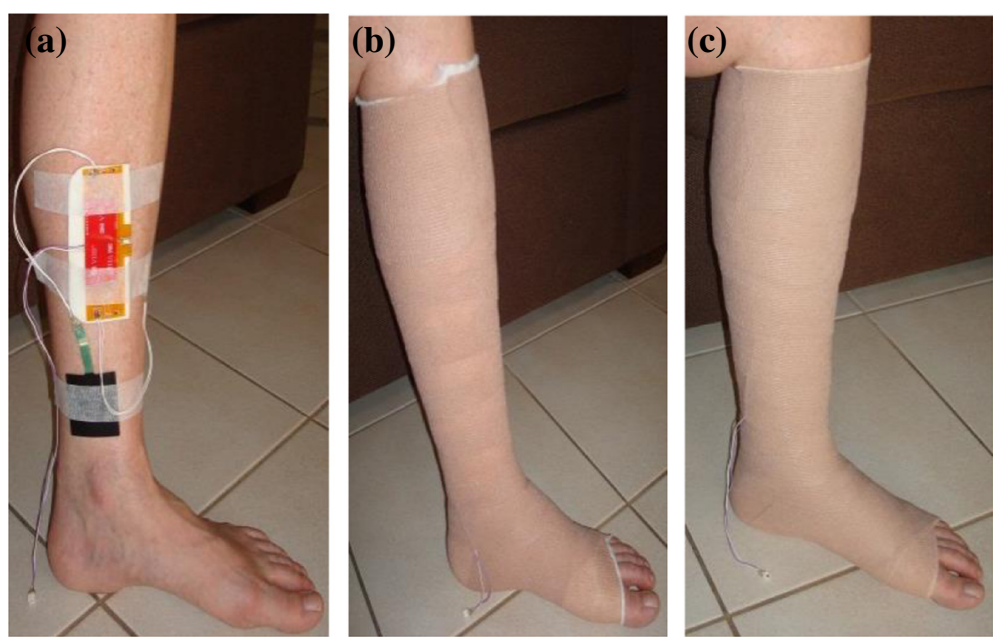

Figure 2 Sensing system under a compression bandage. (a) Sensing system and sensors in place over the leg prior to bandage application (b) 4-layer compression bandaging in place for pressure measurements (c) 2-layer inelastic bandaging in place for pressure measurements. 


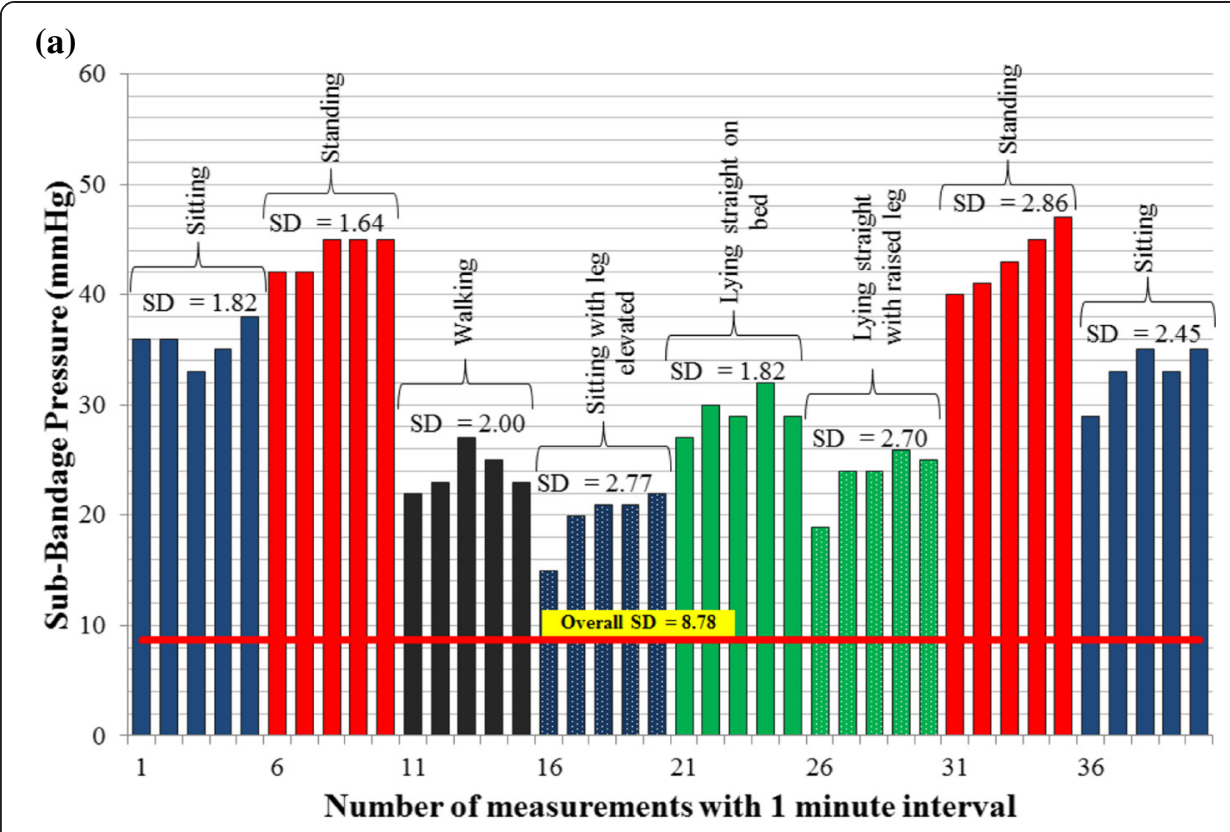

(b)

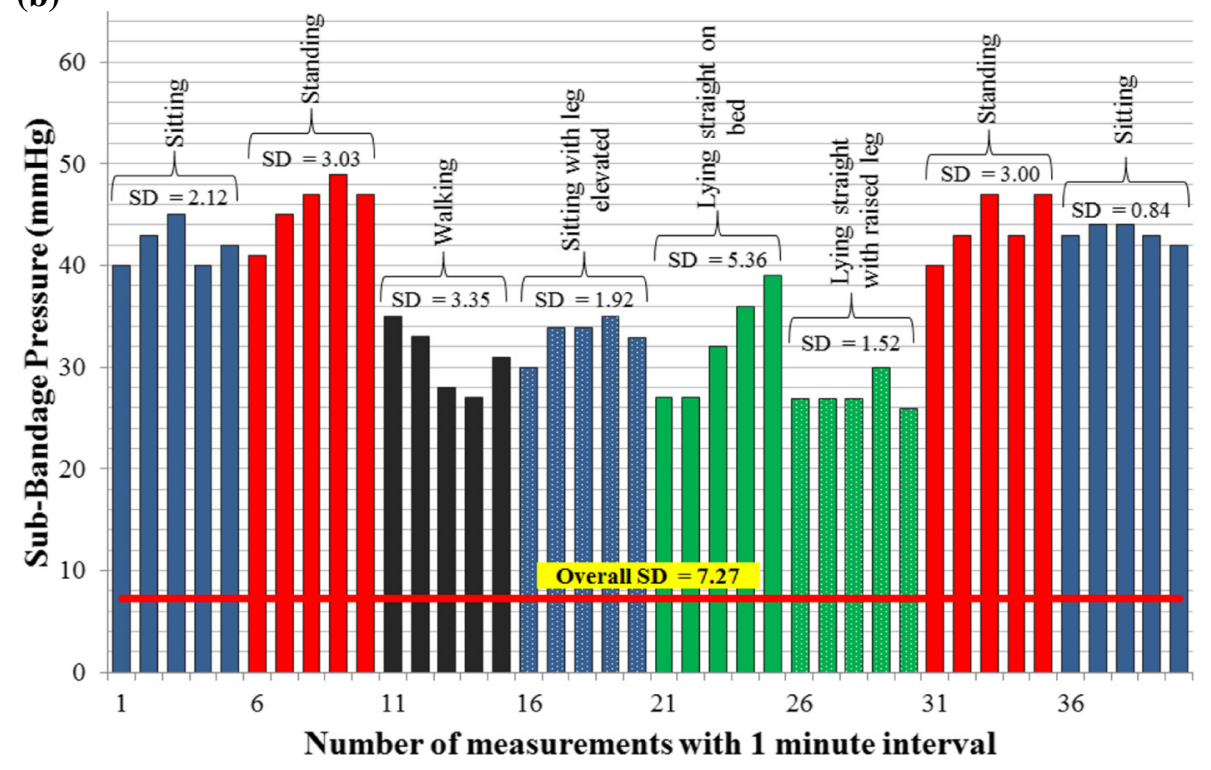

Figure 3 Experimental results of sub-bandage pressure measurements. Graphical plots of pressure measurements during the first trial using (a) 4-layer compression bandage system. (b) 2-layer compression bandage system. The measurements are recorded in various routine movements and postures.

skin with the sensor facing the skin while the pressure sensor was placed over the flat area of the exposed skin, directly above the medial malleolus (ankle), with the sensor facing the skin. Initial measurements were taken in each experiment prior to applying bandages in order to find and nullify any offset readings. The bandages were applied in accordance with the manufacturer's instructions, achieving $40 \mathrm{mmHg}$ starting pressure. The measurement results are plotted in Figure 3(a) (4-layer) and Figure 3(b) (2-layer), at one minute interval using various common postures. Variations in sub-bandage pressure readings are measured by calculating the standard deviation (SD), shown as a red horizontal line in graphs with sub-bandage pressure measurements. 
As the moisture and the temperature measurements are independent of posture changes, different setups were used from those used for sub-bandage pressure measurement. For moisture measurements, a moisture-retentive foam dressing Allevyn ${ }^{\mathrm{Tm}}$ Adhesive (Smith \& Nephew) was used. A small slit was made to insert micro-volume extension set tubing in one corner, and another slit was made to insert the moisture sensor in the opposite corner of the dressing. Since the sensor was dry, the measured moisture level was zero. Fluid was then injected through the tubing after every five minutes until the sensor was soaked and started providing moisture values. After that, the dressing was placed upside down so that the fluid moved away from the sensor until it was completely depleted of moisture. Moisture measurement results are plotted in Figure 4(a).

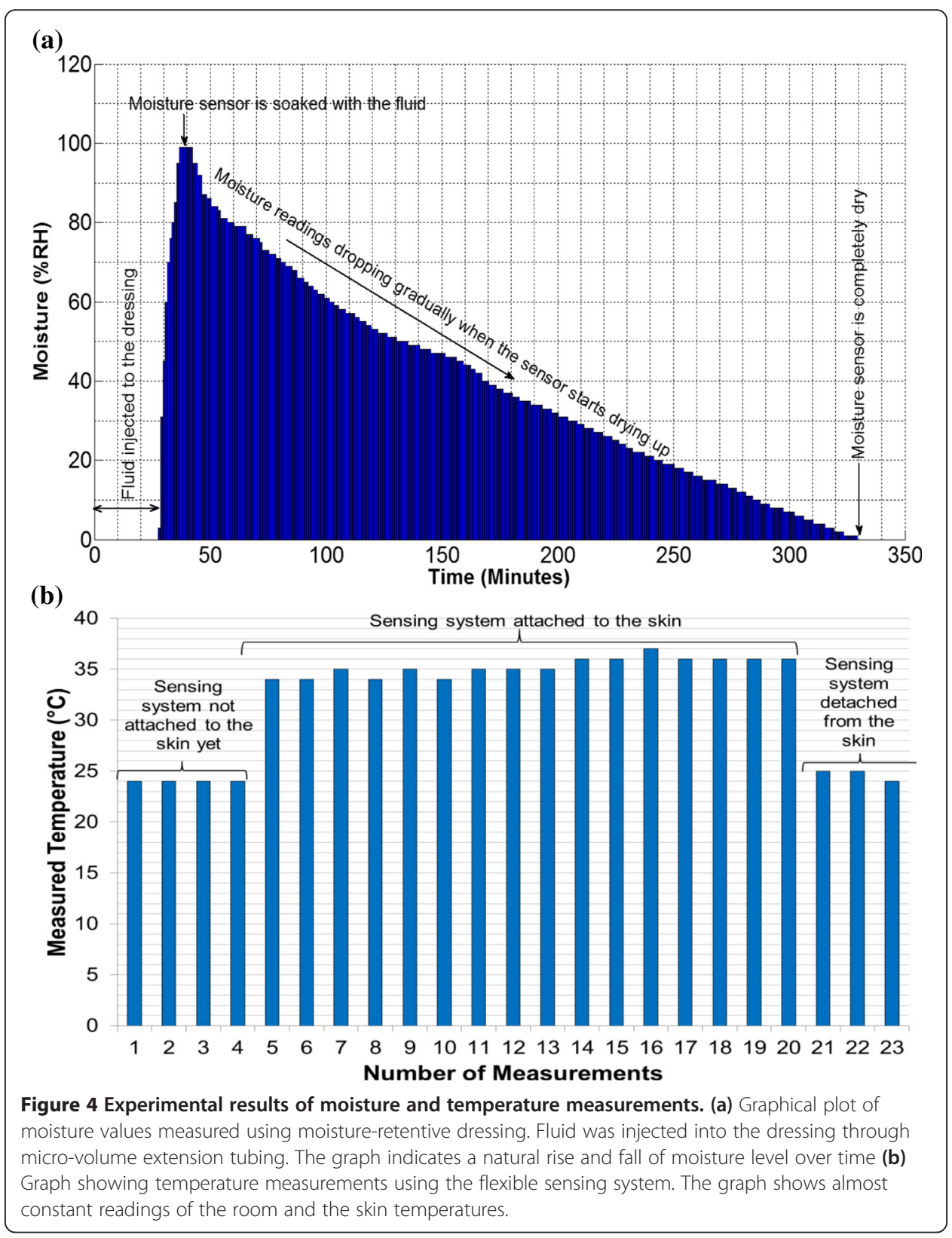


For temperature measurements, the sensing system was placed over the lower leg area with the temperature sensor facing skin, and then various temperature readings were recorded continuously for more than 15 minutes. The experiment was performed at $23^{\circ} \mathrm{C}$ room temperature, and with $40 \%$ humidity. The temperature measurements are plotted in Figure 4(b). The average value of the measured skin temperature was $35 \pm 1^{\circ} \mathrm{C}$ while the standard deviation (SD) was $0.93^{\circ} \mathrm{C}$ for skin temperature measurements.

The sub-bandage pressure measurement results (Figure 3) for both bandages have shown a reliable and repeatable performance of the sensing system under required subbandage pressure. In each posture, five consecutive pressure readings were taken over a period of 5 minutes. For the 4-layer bandage system, the maximum deviation (SD value) observed was $2.86 \mathrm{mmHg}$ during standing, while the same for the 2-layer bandage system was $3.35 \mathrm{mmHg}$ during walking. The moisture level measurement results (Figure 4(a)) have also shown a reliable system performance. A steep rise in moisture level was observed as the injected fluid reached in proximity of the sensor. After that, a gradual and consistent drop in the measured moisture level was observed as the sensor was placed in an upward position to allow backward flow of the fluid. In about six hours, the moisture level dropped to zero when the sensor was completely depleted of the fluid. Thus, the first trial concludes that the measurement of temperature, moisture, and sub-bandage pressure with the developed sensing system is reliable, repeatable and consistent with the changes in experimental conditions.

\section{Measurements at ankle level with a 4-layer bandage}

In this trial, a 4-layer bandage system known to apply $40 \mathrm{mmHg}$ at ankle was used. Bandages were applied as per manufacturer's instructions. A small slit was made in the outermost cohesive bandage (layer 4) to allow battery connection. Allevyn ${ }^{\text {tw }}$ Adhesive (Smith \& Nephew) $12.5 \mathrm{~cm}^{\text {mo }} 12.5 \mathrm{~cm}$ dressing was applied to the lower calf and a small cut was made in the back of the dressing (Figure 5(a)). A micro-volume extension set tubing was attached to the dressing and the slit sealed with film tape to allow injection of fluid (As genuine wound fluid was not available soy sauce diluted with water was used). The fluid was injected under the dressing to mimic wound exudate and soy

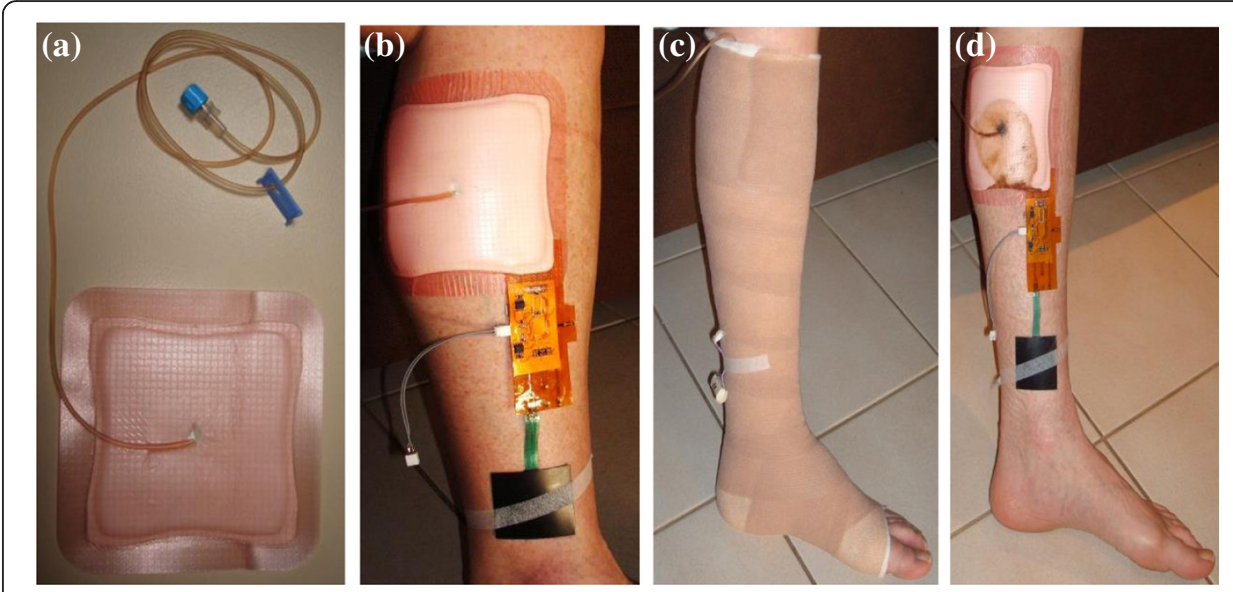

Figure 5 Sensing system under a 4-layer compression bandage. (a) Allevyn ${ }^{\mathrm{TM}}$ dressing with microvolume extension set in place prior to application. (b) Dressing and sensors in place on leg prior to bandage application. (c) Bandaging in place immediately following application. (d) Dressing and sensors in place immediately following removal of bandages. 


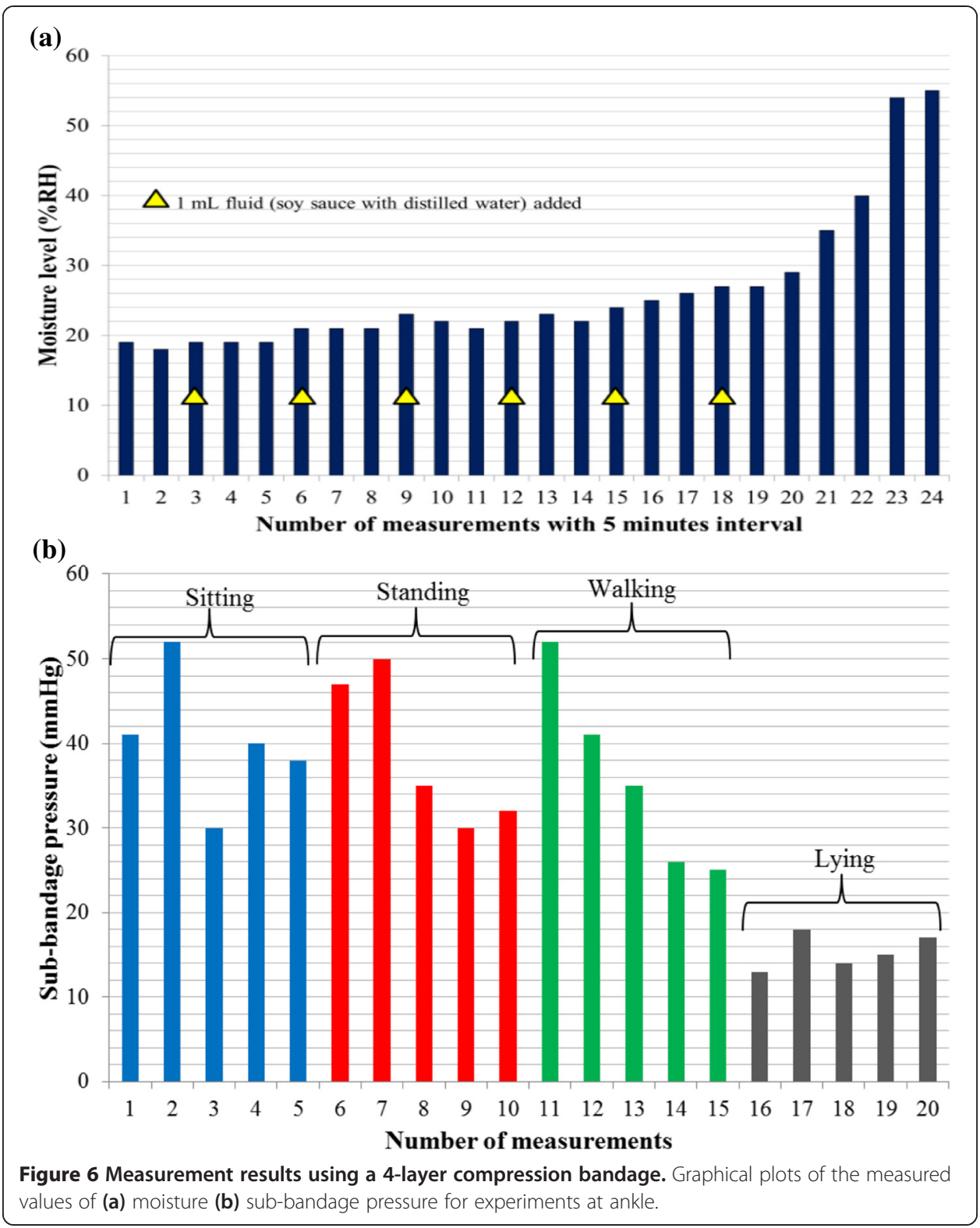

sauce was chosen so that the spread of fluid was visible during experiments. The sensing system was then attached to the lower leg (Figure 5(b)). The pressure sensor was placed proximal to the medial malleolus between exposed skin and bandages. The moisture sensor was placed at the bottom corner of the Allevyn ${ }^{\mathrm{Tw}}$ dressing between the exposed skin and the dressing. The results of second trial are plotted in Figure 6. The average value of temperature during this trial was $33 \pm 1^{\circ} \mathrm{C}$. The moisture values were increasing gradually as more fluid was injected to the dressing until the sensor was soaked with the fluid, the point from where the readings started rising up (readings 21-24 in Figure 6(a)). The sub-bandage pressure values (Figure 6(b)) were dependent on posture, being higher during walking and standing and lower during sitting or lying. It can also be observed from the graph that the pressure readings were consistently dropping over time. This phenomenon can be attributed to loosening of bandage layers with movement. 


\section{Discussion}

In the first trial, the measurements for pressure, moisture, and temperature were taken separately using distinct experimental setups. The measurements were reliable and consistent with the applied conditions as can be observed in Figure 3, Figure 4(a), and Figure 4(b) for sub-bandage pressure, moisture, and temperature respectively. The subbandage pressure values are expected to fluctuate with the movement, and the graphs (Figure 3(a) and (b)) have verified this phenomenon. The overall fluctuation of subbandage pressure values measured as standard deviation was $8.69 \mathrm{mmHg}$ for the 4layer and $7.27 \mathrm{mmHg}$ for the 2-layer bandage system. The postures were changed in a cyclic fashion (i.e. starting from 'sitting' and ending in 'sitting') to isolate the source of fluctuations in measurements.

The average sub-bandage pressure values recorded during the first and last 'sitting' postures for the 4-layer were $35.6 \mathrm{mmHg}$ and $33.0 \mathrm{mmHg}$ respectively for the 4-layer bandage, and were $42.0 \mathrm{mmHg}$ and $43.2 \mathrm{mmHg}$ respectively for the 2-layer bandage. Similarly, the average sub-bandage pressure values during both 'standing' postures were recorded as $43.8 \mathrm{mmHg}$ and $43.2 \mathrm{mmHg}$ respectively for the 4-layer bandage, and were $45.8 \mathrm{mmHg}$ and $44.0 \mathrm{mmHg}$ respectively for the 2-layer bandage. The twin average values during respective postures for each bandage system were pretty close to each other. These measurements proved that the major source of fluctuations was not the sensing system but the movement of the subject. For all other postures including 'walking', 'sitting with leg elevated', 'lying straight', and 'lying straight with raised leg', the bandage pressure dropped significantly because of reduced physical stress on the pressure sensor. The most significant reported drop was almost $58 \%$ of the original value during 'sitting with leg raised' as shown in Figure 3(a). The graphs also indicated increased fluctuations in measurements during certain movements wearing a 2-layer bandage as compared to those with a 4-layer bandage. The variations in measured moisture level (Figure 4(a)) over time verified that the measured values were consistent with the dry, wet and intermediate condition of the moisture sensor. Similarly, the body temperature measurement results were also reliable and stable measuring an average value of $35 \pm 1^{\circ} \mathrm{C}$. Hence, the reliability and consistency of measurements performed on a healthy human volunteer were established during the first trial.

In the second trial, a commonly-used 4-layer bandage system was used to measure sub-bandage pressure near the ankle. The moisture sensor was placed inside a foam dressing through which external fluid was injected in a controlled manner to mimic the wound exudate. This setup was used in this and all following trials. Initial sub-bandage pressure was at $40 \mathrm{mmHg}$. However, sub-bandage pressure was observed to fluctuate with changing postures as observed during the first trial. This variation was caused by many factors including muscle expansion and contraction associated with various postures, changes in blood flow through the muscles as they expand or contract and the loosening of the bandage layers with movement. It can be observed from Figure 6(b) that with continuous movements, the sub-bandage pressure dropped to almost $50 \%$ of the original value. Changes in sub-bandage pressure in such a fashion may adversely affect the healing progress in chronic wounds [43].

In all trials, variations in sub-bandage pressure can be attributed to muscle movement, blood flow direction, and the type and properties of the bandage used. The temperature and moisture measurements did not manifest any dependency on these 
factors. However, the moisture readings may be strongly affected by the combined effect of the gravity pull and the location of moisture sensor with respect to fluid entry point. In a clinical scenario, a consistent rise in measured temperature could mean infection at wound site. A gradual decrease in moisture level might indicate the start of healing and the opposite might mean the worsening of healing state. A consistently increasing sub-bandage pressure could be a sign of infection or excessive swelling in the limb, and a gradual decrease in sub-bandage pressure might mean loosening of the bandage layers.

Using this device, a clinician would be able to visualize the state of healing of a chronic wound remotely without disturbing the wound, a phenomenon which was never possible in chronic wound management before. However, the device needs further miniaturization and performance improvements, such as enhancing measurement resolutions, in order to have a significant impact on chronic wound monitoring. Although, the moisture and temperature sensors could be used for any wound, however, the pressure sensing capability is useful only for venous leg ulcers. The device works with an external battery which needs to be attached at all times. The estimated cost of the proposed sensing system is around $\$ 40$ including sensors $(\$ 10)$, circuit components (\$20), and flexible circuit manufacturing (\$10). This cost could be reduced further if the sensing system is manufactured in bulk quantities and the data is received on a mobile device (i.e. excluding dedicated receiver module). On the other hand, the estimated total cost of the device could potentially exceed the \$40-figure owing to regulatory and constraints of the managing health system. The sensing system and sensors are reusable on a single patient after proper sterilization. The additional cost would be compensated by reduced frequency of dressing changes, reduced nursing time, and reduced use of hospital resources.

\section{Conclusions}

Traditional tools and methods have proven insufficient to effectively monitor the chronic wound healing progress. Inaccuracies in chronic wound measurements are responsible for a significant loss in healthcare budgets e.g. a 30\% loss in the US healthcare budget [2]. Engaging an integrated measurement approach (i.e. measuring other critical parameters [16] in addition to wound dimensions) is believed to be more effective to provide stronger evidence of healing than using one kind of measurement only. Though, the wound area and volume calculations over time, and 3D surveillance techniques are useful for this purpose, the proposed sensing system would be a valuable addition to these approaches to mitigate the losses incurred by human errors in chronic wound measurements.

In this paper, we have presented a novel wireless chronic monitoring system which is flexible, biocompatible, and reliable in performance. The sensing system has been fabricated on a flexible circuit material, enabling it to adapt to human limb contours. Lowpower profile of the sensing device enables it for being operated continuously under a compression bandage over a longer time period (e.g. 3-4 days) without any disturbance to the bandaged wound. Battery life-time can be further enhanced (e.g. 2-3 weeks) by reducing the frequency of wound data transmission. An effective data transmission range of 4-5 m would enable clinicians and nurses to visualize the current wound state from a distance in a hospital environment. 
Experimental results on a healthy human volunteer ascertained that the sensing system was capable of accurately measuring the instantaneous changes in sub-bandage pressure, moisture, and temperature under compression bandages and dressings. Gradual changes in measured wound parameters could reveal the status of healing progress e.g. a consistent rise in sub-bandage pressure might be an indication of infection or swelling. However, the device needs to be tested on patients with chronic wounds to analyze its performance in real environment. The device needs to be tested further even after successful clinical trials to determine its clinical and financial impacts on chronic wound management. The suitability and efficacy of the sensors could be determined during these clinical trials. Nonetheless, the light weight, reliable performance on human limb, flexible and noninvasive structure, low-power consumption and wireless connectivity of the sensing system make it a strong candidate for use in continuous wound sensing and monitoring applications. Future works will incorporate miniaturization of the telemetric sensing system, and its communication with smart phones and display of information on screens using a smart phone application. The system would also incorporate WiFi or 3G communication technologies to share the measured wound parameters to the clinician remotely. This would enable clinicians to gather information on the state of a chronic wound while the patient stays at home.

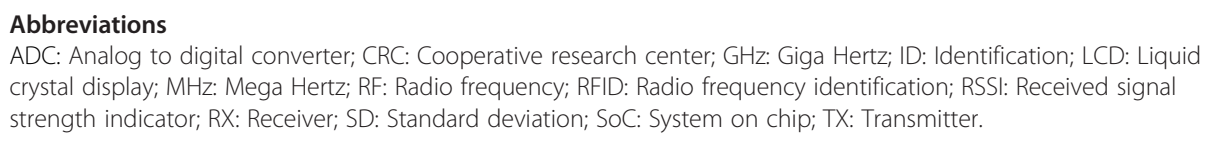

We have been receiving the funding for the current research project from the Wound Management Innovation CRC Australia under Project \# 2.10 since Jan, 2013. The contents in this paper are part of a pending International Patent (PCT) lodged in the Australian Patent Office on 20th Oct, 2014.

\begin{abstract}
Authors' contributions
NM drafted the manuscript, carried out the literature study, participated in the design and testing of the whole system, and performed theoretical and statistical analysis. AH contributed in conception of the project, contributed in critical analysis of theory and results, and reviewed and revised the manuscript critically. ST participated in experimental study, carried out the experiments, and reviewed and edited the manuscript. NV contributed in conception of the project, conceived of experimental strategy, contributed in critical analysis and interpretation of results, reviewed and revised the manuscript critically. All authors read and approved the final manuscript, and agreed to be accountable for all aspects of the work.
\end{abstract}

\title{
Acknowledgements
}

We are thankful to the Wound Management Innovation Cooperative Research Centre (CRC) Australia for sponsoring this work.

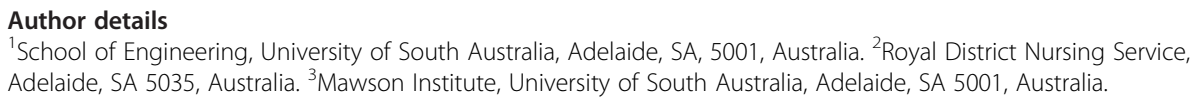

1. Cadogan J, Baldwin D, Carpenter S, Davey J, Harris H, Purser K, et al. Identification, diagnosis and treatment of wound infection. Nurs Stand. 2011;26:44-8.

2. Nixon Mark MD, Christine M. "Evidence-Based Wound Surveillance-A Three-Dimensional Approach To Measuring, Imaging and Documenting Wounds," Aranz Medical 2014.

3. The Economic Cost of Wounds. 2014. Available: http://www.smith-nephew.com/about-us/what-we-do/advancedwound-management/economic-cost-of-wounds/.

4. Wound Management Innovation CRC. 2013. Available: http://www.woundcrc.com/mediareleases.html.

5. Harding KG, Edwards R. Bacteria and wound healing vol. 17. United States: Lippincott Williams \& Wilkins; 2004

6. Menke NB, Ward KR, Witten TM, Bonchev DG, Diegelmann RF. Impaired wound healing. Clin Dermatol. 2007;25:19-25.

7. Dipietro LA, Guo S. Factors affecting wound healing vol. 89. United States: Sage Publications Inc; 2010.

8. Higher wound care costs are driving treatment research (McKnight's Long-Term Care News, published in Jun, 2012 Issue). 2014. Available: http://www.mcknights.com/higher-wound-care-costs-are-driving-treatment-research/article/244578/. 
9. Abdelrahman T, Newton H. "Wound dressings: principles and practice". Surgery (Oxford). 2011;29:491-5

10. Partsch H, Clark M, Mosti G, Steinlechner E, Schuren J, Abel M, et al. "Classification of compression bandages: practical aspects". Dermatol Surg. 2008;34:600-9.

11. Fletcher A, Cullum N, Sheldon TA. A systematic review of compression treatment for venous leg ulcers. Br Med J. 1997;315:576.

12. Hopkins A. "How to apply effective multilayer compression bandaging." 2006. Wound Essentials (Wounds UK), vol. 1.

13. Kumar B, Das A, Alagirusamy R. Analysis of sub-bandage pressure of compression bandages during exercise. J Tissue Viability. 2012;21:115-24.

14. Ovington LG. Advances in wound dressings. Clin Dermatol. 2007;25:33-8.

15. Lagana G, Anderson EH. "Moisture dressings: the new standard in wound care". J Nurse Pract. 2010;6:366-70. 5.

16. Harding K. Diagnostics and wounds. World Union of Wound Healing Societies (WUWHS): A consensus document; 2007.

17. Dargaville TR, Farrugia BL, Broadbent JA, Pace S, Upton Z, Voelcker NH. "Sensors and imaging for wound healing: a review. Biosensors Bioelectronics. 2013:41:30-42.

18. Matzeu G, Losacco M, Parducci E, Pucci A, Dini V, Romanelli M. "Skin temperature monitoring by a wireless sensor," in IECON 2011 - 37th Annual Conference on IEEE Industrial Electronics Society, 2011, pp. 3533-3535

19. Moser Y, Gijs Martin AM. Miniaturized flexible temperature sensor. J Microelectromech Syst. 2007;16:1349-54.

20. McColl D, Cartlidge B, Connolly P. Real-time monitoring of moisture levels in wound dressings in vitro: An experimental study. Int J Surg. 2007:5:316-22.

21. Ohmedics Ltd. WoundSense product information. 2014. Available: http://www.ohmedics.com/resources/IFU3+ Eng+A6+v1.1+Aug+11.pdf.

22. Al Khaburi J, Dehghani-Sanij A, Nelson EA, Hutchinson J et al. "Pressure mapping bandage prototype: Development and testing," in Biomedical Engineering (ICoBE), 2012 International Conference on, 2012, pp. 430-435.

23. Casey V, McAree B, Moloney MC, Grace P. "Wearable sub-bandage pressure measurement system," in Sensors Applications Symposium (SAS), 2010 IEEE, 2010, pp. 41-45.

24. Codman Microsensor and ICP Transducer. 2014. Available: http://emea.depuysynthes.com/hcp/codman-neuro/ products/qs/icp-monitoring-system.

25. Mejzlik J. Use of a silicon microsensor for pressure monitoring inside the wound and outcomes on healing. J Wound Care. 2012:21:589.

26. Xue N, Chang SP, Lee JB. A SU-8-based microfabricated implantable inductively coupled passive RF wireless intraocular pressure sensor. J Microelectromech Syst. 2012;21:1338-46.

27. Sauser FE, Glos D, Bylski-Austrow D, Papautsky I. "Pressure sensors for in vivo measurements on spinal growth plates," in Engineering in Medicine and Biology Society, IEMBS '04. In: 26th Annual International Conference of the IEEE, 2004. 2004. p. 2430-3

28. Clausen I, Moe ST, Tvedt LGW, Vogl A, Wang DT. "A miniaturized pressure sensor with inherent biofouling protection designed for in vivo applications," in Annual International Conference of the IEEE Engineering in Medicine and Biology Society, United States, 2011

29. Hasenkamp W, Forchelet D, Pataky K, Villard J, Lintel HV, Bertsch A, et al. Polyimide/SU-8 catheter-tip MEMS gauge pressure sensor. Biomed Microdevices. 2012;14:819-28.

30. Hill GC, Melamud R, Declercq FE, Davenport AA, Chan IH, Hartwell PG, et al. SU-8 MEMS Fabry-Perot pressure sensor. Sensors Actuators A Phys. 2007;138:52-62.

31. DeHennis $A D$, Wise KD. "A wireless microsystem for the remote sensing of pressure, temperature, and relative humidity." 2005; 14.

32. Schlierf R, Horst U, Ruhl M, Schmitz-Rode T, Mokwa W, Schnakenberg U, et al. A fast telemetric pressure and temperature sensor system for medical applications. J Micromech Microeng. 2007;17:S98-S102.

33. Mehmood N, Hariz A, Fitridge R, Voelcker NH. "Applications of modern sensors and wireless technology in effective wound management." J Biomed Mater ResPart B: Appl Biomater, pp. n/a-n/a, 2013.

34. Mehmood N, Hariz A, Templeton S, Voelcker NH. "An Innovative Sensing Technology for Chronic Wound Monitoring," in Australian Biomedical Engineering Conference, Canberra, Australia, 20-22. 2014

35. Mehmood N, Hariz A, Templeton S, Voelcker NH. Calibration of sensors for reliable radio telemetry in a prototype flexible wound monitoring device. Sensing Bio-Sensing Res. 2014;2:23-30.

36. Ochoa M, Rahimi R, Ziaie B. "Flexible sensors for chronic wound management". Biomed Eng, IEEE Rev. 2014;7:73-86.

37. Online datasheet Texas Instruments temperature sensor LM94021. 2013. Available: http://www.ti.com/lit/ds/ symlink/Im94021.pdf.

38. Online datasheet of RF transceiver ATMega128RFA1. 2013. Available: http://www.atmel.com/devices/ ATMEGA128RFA1.aspx.

39. Online datasheet of RF Balun 2450FB15L0001E by Johanson Technology. 2013. Available: http://www. johansontechnology.com/datasheets/balun-filter/2450FB15L0001.pdf.

40. Online datasheet of $2.4 \mathrm{GHz}$ chip antenna model 2450AT43B100 by Johanson Technology. 2013. Available: http:// www.johansontechnology.com/datasheets/antennas/2450AT43B100.pdf.

41. Online datasheet of DS-G160128STBWW LCD module. 2013. Available: https://www.sparkfun.com/datasheets/LCD/ DS-G160128STBWW.pdf.

42. Online datasheet of Toshiba T6963C dot matrix LCD controller. 2013. Available: http://www.mikroe.com/ downloads/get/1910/t6963c_spec.pdf.

43. Keller A, Müller ML, Calow T, Kern IK, Schumann H. Bandage pressure measurement and training: simple interventions to improve efficacy in compression bandaging. Int Wound J. 2009;6:324. 